\title{
Synthesis of self-assembled nacre-like materials with 3D periodic layers from nanochitin via hydrogelation and mineralization
}

Junhua Xu

Nanjing Forestry University

Liang Liu

Nanjing Forestry University https://orcid.org/0000-0002-5430-2501

Juan Yu

Nanjing Forestry University

Yujun Zou

Nanjing Forestry University

Wenhui Pei

Nanjing Forestry University

Lili Zhang

Nanjing Forestry University

Wenbo Ye

Nanjing Forestry University

Long Bai

University of British Columbia

Zhiguo Wang

Nanjing Forestry University

Yimin Fan ( $\sim$ fanyimin@njfu.edu.cn )

Nanjing Forestry University https://orcid.org/0000-0003-2764-1310

Qiang Yong

Nanjing Forestry University

Orlando Rojas

University of British Columbia

\section{Article}

Keywords: self-stratification, hydroxyapatite, nanochitin, biomineralization, periodic layering, hydrogelation

Posted Date: February 25th, 2021 
DOI: https://doi.org/10.21203/rs.3.rs-239873/v1

License: (c) (1) This work is licensed under a Creative Commons Attribution 4.0 International License. Read Full License 


\section{Abstract}

Here introduced a route for the synthesis of 3D structures that display a mechanical strength that competes with that of the toughest materials found in nature. Following the "brick-and-mortar" biomineralization typical of nacre, self-stratified, periodic materials are obtained by one-step ion diffusion gradient and hydrogelation of nanochitin with simultaneous mineral coprecipitation. Specifically, under appropriate electrolyte conditions, hydroxyapatite (HA) microspheres grow in an organic network formed from partially deacetylated chitin nanofibers ( $N C h)$, resulting in periodic stacking of mineralized $(\mathrm{HA})$ and non-mineralized (NCh) layers. By directional diffusion, customizable 3D shapes are self-assembled and demonstrated to function as optical waveguides with selective light transmission at interfaces. Upon hot pressing, the resulting solid structures exhibit a superb mechanical performance while being biocompatible (tested with chondrogenic ATDC5 cells as a model for physiological mineralization). Overall, a shape-controlled, one-pot biomineralization method was proposed that achieves hierarchical, periodic and strong "nacre-like" structures suitable as biomedical material.

\section{Introduction}

Biogenic materials usually exceed the mechanical performance achievable by man-made counterparts, even if built from similar components. This justifies the ongoing quest to approach biomimicry as a design process to achieve such performance, ${ }^{1-6}$ which has considered biomineralization and other mechanisms. In the animal kingdom, such processes lead to the formation of bones, enamel and cuticles, among others. ${ }^{7,8}$

Nacre, a "gold model" for biomineralization, is a typical biomineral material that displays a highly ordered, multilevel hierarchical structures. ${ }^{9}$ In nacre, brittle flakes of calcium carbonate ( 95\%) bind to a biopolymer component $(\sim 5 \%)$ to form alternating organic and inorganic layers, ${ }^{3}$ e.g., a "brick-and-mortar" (BM) structure that displays an extraordinary strength and fracture toughness. ${ }^{10}$ The excellent properties of such structures depend on the inherent supramolecular assembly, typically spanning multiple size scales. Natural nacre is a perfect example of the genetically-determined synergy between organic and inorganic components.

Nacre formation is distinctively slow, since it takes years to complete; therefore, provided that a similar performance is achieved, the possibility of faster processing is an inviting proposition. So far, the synthesis of nacre-like BM structures have considered (a) layer-by-layer deposition, ${ }^{11-14}$ (b) selfassembly, ${ }^{15-18}$ and (c) freeze-casting. ${ }^{19-22}$ In layer-by-layer deposition, solvent volatilization or filtration is firstly used to produce sheet-like inorganic structures (nanoscale MTT, graphene oxide, HA, nanoclay), which are then combined with an organic phase (PVA, chitosan, silk, alginate). ${ }^{23-30}$ The resulting nanosheets resemble native nacre but suffer from the uncontrollable assembly of the organic phase. ${ }^{24,27}$ The first step in freeze-casting or templating involves the formation of an organic lamellar framework that is then filled with the inorganic component, representing a large number of possible permutations. ${ }^{31}$ 
Recently, organic-inorganic composites have been proposed by co-precipitation. ${ }^{32,33}$ Unfortunately, controlling the orientation and pattern design have remained as major challenges, which is in contrast to the hierarchical and ordered structures that form naturally in nacre.

In an attempt to address the challenges of producing synthetic nacre, we introduce partially deacetylated chitin nanofibers ( $\mathrm{NCh}$ ) as building blocks that develop cationic charges in aqueous suspension and are pH-responsive, allowing controlled gelation. Taking advantage of such properties and inspired by nature, we use NCh in organic templating and biomimetic mineralization (Supplementary Fig. 1). ${ }^{34}$ By mixing mineral ions and hydrogelation through evolution of ammonia gas, self-stratified hierarchical and periodic layers are formed from hydroxyapatite (HA) and the NCh hydrogel. Ultimately, HA-NCh dehydration followed by hot pressing leads to nacre-like 3D materials that display a remarkably high mechanical strength (Fig. 1a), comparable to that of natural nacre and surpassing those of relevant manmade materials.

\section{Results And Discussion}

Formation of self-stratified, periodic layers of HA-NCh hydrogel $(\mathrm{LH})$. Crab shell a-chitin is highly crystalline, which potentially fibrillar systems ideally suited for mineral deposition. NCh was stably dispersed under acidic conditions and further assembled into networks by $\mathrm{pH}$ shifting. ${ }^{35} \mathrm{The} \mathrm{pH}$ responsiveness of NCh and its gelation were synergistically combined with hydroxyapatite (HA) minerals, making an ideal system for coprecipitation. ${ }^{36,37}$ In this work, we used gradient hydrogelation and mineralization in one-pot, self-stratifying systems. Electrolytes comprising $\mathrm{Ca}^{2+}$ and $\mathrm{PO}_{4}{ }^{3-}$ ions $(\mathrm{Ca} / \mathrm{P}$ ratio of 1.67), were added to an aqueous nanofiber suspension, followed by ultrasonic homogenization, increasing the viscoelasticity of the system by ionic crosslinking and endowing the gels with high levels of conformability when cast in molds of given shapes (Fig. 1b, Supplementary Fig. 2). Furthermore, ammonia-based gas phase gelation allowed the formation of a layered HA mineral structure in the presence of the organic NCh phase (Supplementary Videos 1 and 2).

Following the above process, layered HA-NCh hydrogels (herein referred to as "LH") were self-assembled into multiscale, ordered and periodic structures (Fig. 1c-h). Their smallest characteristic dimension corresponded to HA nanosheets ( $1.5 \mathrm{~nm}$ thickness, Supplementary Fig. 3) stacked with NCh (diameter of $\sim 10 \mathrm{~nm}$ and hundreds of nanometers in length, Supplementary Fig. 1). The mineral content in the network reached values as high as 60.6\% (dry weight) (Supplementary Fig. 4), which is otherwise not reachable in natural crab or shrimp shells. More specifically, HA microspheres were held in a NCh network, which presented pores of only tens to hundreds of nanometers in size (Supplementary Fig. 5). At larger scales, HA-NCh composites (mineralized layer rich in HA) and pure NCh layers (non-mineralized, with no HA) stacked alternately, forming a periodic LH of macroscopically stratified structures. The distribution of the elements on the surface (Fig. 1(i-l) and Supplementary Fig. 6) indicated calcium and phosphorus present mainly in the HA microspheres, distributed in the mineralized layers. By contrast, the nonmineralized layers were composed of carbon and oxygen species, depleted in phosphorus and calcium. 
The results further established that HA was distributed in the gel following a regularly stratified pattern. Complementary analyzes (FTIR, XRD and XPS, Supplementary Fig. 7-9) confirmed the presence of HA in the mineral phase. ${ }^{38}$

Stratification mechanism. HA formation took place under the increased $\mathrm{pH}$ caused by ammonia diffusion in the gas phase. Nucleation of $\mathrm{HA}, \mathrm{Ca}^{2+}$ and $\mathrm{PO}_{4}{ }^{3-}$ occurred at a threshold $\mathrm{pH}$, forming crystals in the organic network. Simultaneously, deprotonation of NCh's surface amino groups took place (the organic phase), thereby reducing the electrostatic repulsion between the nanofibers and consolidating a hydrogen-bonded network. ${ }^{39}$ Gelation and mineralization occurred consecutively, forming a strong LH. Most remarkably, the mineral phase was arranged as a macroscopic, layered and highly ordered structure interlaced with the organic phase. The spontaneous and autonomous features of the process, reported here for the first time, departs from the multilayer structures that are typically formed in a stage-wise manner.

As indicated in Fig. 2a, the mineralized, periodic and multilayered structures after an induction step. The latter occurs at the onset of HA formation, as a homogeneous gel (Fig. 2a and Supplementary Fig. 10). After a given time, stratified gel is formed spontaneously (Supplementary Fig. 11), as a result of ammonia mass diffusion in the gel via Fick's second law, as a function of a concentration gradient. ${ }^{40}$ Accordingly, for a given initial concentration, the molecular diffusion rate decreases with time. On the other hand, according to Henry's law, the partial pressure of ammonia in the gas phase is constant (isothermal conditions). ${ }^{41}$ Thus, ammonia concentration at the surface of the aqueous phase (hydrogel) is also constant. Overall, in the early stage of mineralization, the transfer rate of ammonia is expected to be very fast but gradually decreases with the progression of time. This process induces HA precipitation and NCh crosslinking following a mechanism that leads to macroscopically-ordered layered structures, under "ion diffusion and gradient gelation" (IDGG) (Fig. 2a, b).

In sum, we hypothesize that LH formation involves (1) the growth of an uniform HA-NCh hydrogel layer when the transfer rate of ammonia, $V_{A M}$, is greater than that of mineral ions, $V_{M I}\left(V_{A M}>V_{M I}\right)$. Following, (2) self-stratification of the hydrogel occurs forming a periodic pattern when $V_{A M}<V_{M I}$. In this latter stage, ammonia and mineral ions firstly reach a nucleation concentration (NC), leading to the formation of $\mathrm{HA}$ nuclei. Subsequently, neighboring ions diffuse to the site to support HA growth into a mineralized layer. Simultaneously, ion depletion takes place underneath the mineralized layer. The nucleation and mineralization are prevented if the ions concentration is much lower than NC. In sum, lamellar or layered mineralization occurs by simultaneous mineralization and ion diffusion, following a top-down evolution, from the air/water interface and progressively growing until reaching the deepest regions of the system. The proposed LH formation process is depicted in Fig. 2c.

Diffusion of alkali ammonia vapor produces a pH gradient in the out-of-plane direction (top-down), while a uniform $\mathrm{pH}$ is maintained at the given layer (latitudinal level). When the $\mathrm{pH}$ rises to a value corresponding to $\mathrm{HA}$ nucleation (Fig. 2b), $\mathrm{Ca}^{2+}$ and $\mathrm{PO}_{4}{ }^{3-}$ form $\mathrm{HA}$ crystal nuclei, thereby continuously 
followed with HA mineralization, forming a mineralized layer. Simultaneously, during HA mineralization a $\mathrm{pH}$ shift occurs: $\mathrm{OH}^{-}$ions are consumed, leading to a local $\mathrm{pH}$ reduction. Along this process and underneath the mineralized layer, a neighboring organic layer is formed by non-mineralized ions that are continuously consumed, forming a gradient of reduced ion concentrations. The ions are constantly transferred to the mineralized layer through ionic diffusion and form an ion-defect layer, depleted in $\mathrm{Ca}^{2+}$ and $\mathrm{PO}_{4}{ }^{3-}$. This is a reason why $\mathrm{HA}$ microspheres form at the bottom of the mineralized layer, with sizes that are much larger than those formed at the upper levels (Fig. 1f-h). Accordingly, a non-mineralized layer is formed. In the ionic diffusion area, the concentrations of $\mathrm{Ca}^{2+}$ and $\mathrm{PO}_{4}{ }^{3-}$ increase gradually, in the downward direction, until reaching another high $\mathrm{Ca}^{2+}$ and $\mathrm{PO}_{4}{ }^{3-}$ concentration (Fig. $2 \mathrm{~b}$ ). With the progression of time, ammonia continues to diffuse downwardly until reaching an ion concentration that leads to new nucleation, at a certain distance below the last mineralization layer. Thus, repeated cycles take place, involving nucleation and mineralization, formation of mineralized and defect layers, eventually forming macro-layered periodic structures.

LH structuring control. As noted before, LH is formed by IDGG, uniformly and following a self-assembly process. Here we show that the structure can be finely controlled, for example, by adjusting the ammonia concentration through the volume of liquid ammonia (Supplementary Fig. 12). For instance, the mineralization rate is very slow at low ammonia concentrations, but stratification occurs at the early stages. To highlight the role of ammonia diffusion in the stratification process, we conducted additional experiments where ammonia was replaced with $\mathrm{NaOH}$. The latter specie was expected to produce a pH shift and enable nucleation and mineralization of HA in the NCh network. However, this was not the case since $\mathrm{NaOH}$ failed to trigger the formation of stratified structures.

Ammonia is a weak alkali and, contrary to aqueous $\mathrm{NaOH}$, it is volatile ${ }^{42}$ and gradually volatilizes and enters in contact with the aqueous NCh and $\mathrm{Ca} / \mathrm{P}$ mixture; thus, the permeation and diffusion of ammonia continuously proceeds in the downward direction, which leads to a $\mathrm{pH}$ or ion concentration gradient. By contrast, $\mathrm{NaOH}$ directly dissociates into $\mathrm{OH}^{-}$ions that once in contact distribute in the $\mathrm{NCh}$ and $\mathrm{Ca} / \mathrm{P}$ system, preventing a gradual relaxation and permeation. Therefore, along with the $\mathrm{pH}$-induced mineralization and gelation, a gradient diffusion is necessary for self-stratification. Moreover, IDGG allows the formation of mineralized HA-NCh hydrogel in a tailorable fashion, allowing the control of the stratification (Fig. 3), as well the shape, pattern, layer thickness and structure. Given that ammonia diffusion occurs in the downward direction, when using a cylindrical vessel, the ammonia gas contacts the upper surface, and diffuses evenly top-down. This leads to HA-mineralized layers that stack parallel to the upper surface. By controlling the direction of ammonia diffusion, more complex patterns can be designed, depending on the shape of the mold and ammonia transport.

HA layers in the LH can form in triangular or circular "onion" patterns (Fig. 3b). Other designs are possible with a layer thickness and structure that is controlled with the concentration of $\mathrm{Ca}^{2+}$ ions and the temperature (or by using regulating species, such as ethanol, Fig. 3a). Indeed, higher precursor 
concentrations and mineralization temperatures, and the addition of ethanol, resulted in a denser layered HA structure.

The above observations cannot be directly rationalized in terms of the rate of ion transfer; the HA mineralization process should be considered. In fact, we found that the layer thickness was linearly related to the $\mathrm{pH}$ of $\mathrm{HA}$ nucleation (Supplementary Fig. 13) in which a denser lamellar structure was formed at lower nucleation $\mathrm{pH}$. All in all, the LH prepared under the proposed mechanism developed a grating-like, periodic structure, with a layer thickness that can be tailored, from tens of microns to few millimeters.

The structure of the gel included an opaque HA inorganic mineral interlaced with a transparent, organic NCh network. Such periodic structures showed interesting selective optical reflection: light transmission followed the path parallel to the mineralized layer, acting as a waveguide, with the light being blocked in the direction perpendicular to the mineralized layer (Fig. 3c, Supplementary Video 3). It is important to note that HA was distributed in the gel network as a periodic lamellar structure, while the NCh network was evenly distributed throughout the gel.

Synthesis of "nacre-like" HA-NCh layered plates (LP). So far, the obtained hybrid composite structures are similar to those found in nacre or crab shells, which involve biological minerals, proteins and pigments (but with much less water). Importantly, a much shorter time was needed in our process. Compared to any other reported approach, the one-pot, rapid and customizable synthesis are important attributes of our proposed protocol. Here, the hybrid stacking of $\mathrm{LH}$ were further advanced into nacre-like layered HANCh solid plates (herein termed as “LP”).

Freeze-dried dehydration followed by hot pressing was applied to obtain nacre-like LP (Fig. 4a, d) with a remarkable mechanical strength. We noticed a "brick-and-mortar" (BM) structure following closely the hierarchical assembly of natural abalone. It was observed that on the top surface of the "nacre-like" LP, HA beads were evenly distributed and separated by organic matter, similar to the Taylor polygonal structure of aragonite in abalone (Fig. 4b, e) ${ }^{3}$ The BM structure was formed by alternating stacking of organic and inorganic components. Moreover, a step-like staggered structure was found on the oblique fracture surfaces of the LP, similar to that of natural abalone shells (Fig. 4c, f). The mineral layers formed by HA were approximately $10 \mu \mathrm{m}$, and the organic chitin layers were approximately $200 \mathrm{~nm}$ (Fig. 4j-n).

Following the removal of inorganic HA layers by acid treatment, the remaining NCh organic layers were compacted (Supplementary Fig. 14). The assembled structures and organic binders led to energy dissipation, which is the structural basis for the development of the macroscopic properties of the synthesized LP. Indeed, upon fracture, a significant crack deflection was observed, forming a zig-zag crack pattern and interlaminar delamination, Fig. $4 \mathrm{~g}$-i. The multilayer nacreous structure led to crack deflection, crack branching and splitting, which are key mechanisms that explain the strength and toughness of our synthesized LP, similar to that of natural pearl. 
The LP and its alternating organic and inorganic components, each with respective mechanical strength, contributed to a segmented crack surface, which was tested by surface nanoindentation. As expected, the mineralized HA layer had a higher modulus, given its inorganic nature. By contrast, the modulus of the organic layer was lower. The cracked surface of the whole sample showed alternating "high-low" mechanical strength (Fig. 5a).

Three-point stress-strain curves of specimens containing different amounts of HA, namely, LP-0.25, LP0.5 and LP-1 (corresponding to original Ca addition of $0.25 \%, 0.5 \%$ and $1 \% \mathrm{wt} / \mathrm{v}$, respectively), are displayed in Fig. 5b. HA is a hard and brittle material that plays an important role in the strength of the sample but has a negative effect on toughness.

The mechanical properties of pearls have been extensively reported. ${ }^{30,43}$ Herein, we compared our results with those of four natural nacres, abalone, oyster, mussel and pectinidae (Fig. 5c, Supplementary Fig. 15). The mechanical properties of our LP are similar to those of natural pearly materials (Supplementary Table 1). We noted that while LP is homogeneous at the macro- or sub-microscopic levels, few unavoidable defects were present in the microscale. Owing to such defects, the specific fracture stress and specific elastic modulus were slightly smaller than those of natural pearls, cortical bone or a few artificial materials (Fig. 5d). ${ }^{12,31,38,44-49}$ Nevertheless, the prepared LP displayed a mechanical strength that surpassed that of cancellous bone. It also showed a better elastic modulus than that synthetic MTT/CNF composites. Notably, and as discussed, the composites synthesized in this study displayed multilevel and controllable hierarchical structures. Finally, considering the application of LP as a biomaterial, we studied its biocompatibility with ATDC- 5 cells (chondrogenic ATDC 5 cells used as a model for physiological matrix mineralization). The results (Supplementary Fig. 16) showed that cells adhered and proliferated on LP, indicating its excellent prospects as substitute for artificial bone and related biomaterials.

\section{Conclusions}

We introduced organic-inorganic composite hydrogels synthesized by ion diffusion and gradient gelation. The process involves rapid and spontaneous stratification into layers, under no external control. The composites showed HA distributed in regularly interspaced layers, forming periodic patterns that could be selected by design. The mineralized, multiscale hydrogels, comprising alternating organic-inorganic structures, showed selective light transmission and direction-dependent properties. They were further processed into plates showing BM structures, similar to those that occur in nature, e.g., nacre or $\mathrm{crab} /$ shrimp shells. Upon mechanical loading, the fractured areas observed in the cross section of the plates indicated alternating strong-weak zones, as expected from layering. This is likely a key factor explaining the high strength and toughness of the material. The combination of simple preparation, customizable formation, strength and biocompatibility make the HA-NCh system a biomaterial suitable for scaffolding or as bone substitute. Our biomaterial-inspired designs open the possibilities for easy manufacturing of strong, multiscale materials that are otherwise not accessible using currently available fabrication protocols. 


\section{Materials And Methods}

Preparation of partially deacetylated nanochitin (NCh). Purification of chitin: The chitin used in our experiments was purified from the shells of crabs (Eriocheir sinensis). Briefly, the shells were first immersed in $1 \mathrm{M} \mathrm{HCl}$ for $12 \mathrm{~h}$ to remove minerals, and then immersed in $1 \mathrm{M} \mathrm{NaOH}$ for $12 \mathrm{~h}$ to remove protein. This process was repeated three times to make sure the protein and mineral were removed completely. The pigment was bleached by treatment with $0.5 \% \mathrm{wt} / \mathrm{v} \mathrm{NaClO}{ }_{2}$ at $\mathrm{pH} 5,70^{\circ} \mathrm{C}$ for $2 \mathrm{~h}$. The purified chitin was obtained after washing with distilled water. ${ }^{34}$

Preparation of NCh: The NCh was prepared by partially deacetylation and mechanical treatment. Briefly, 4 $\mathrm{g}$ of purified chitin was dispersed in $200 \mathrm{ml}$ aqueous $\mathrm{NaOH}$ (35\% wt/wt) under continuous stirring in water bath at $90^{\circ} \mathrm{C}$ for $4 \mathrm{~h}$. After washing with distilled water, partially deacetylated chitin with degree of deacetylation (DDA) of $\sim 26 \%$ was obtained. For preparation of chitin nanofibers, $1 \mathrm{~g}$ of partially deacetylated chitin was dispersed in $100 \mathrm{ml}$ aqueous acetic solution (1\% wt/v), followed by high pressure homogenization at 500 bar, 5 passes. A NCh dispersion of 1\% wt/v concentration was obtained.

Fabrication of HA-NCh hydrogels $(\mathrm{LH})$ with layered mineralized structure. Preparation of ion precursor buffer: Solution A, $5 \mathrm{~g}$ of $\mathrm{CaCl}_{2}$ was dissolved in $15 \mathrm{ml} 1 \mathrm{M} \mathrm{HCl}$. Solution B, $5.7 \mathrm{~g}$ of $\mathrm{NaH}_{2} \mathrm{PO}_{4} \cdot 12 \mathrm{H}_{2} \mathrm{O}$ was dissolved in distilled water. Then solution A was mixed with solution B slowly under constant stirring. The molar ratio of $\mathrm{Ca}$ and $\mathrm{P}$ was 1.67, which is the same to that of $\mathrm{HA}$. The co-precipitation reaction is as follow:

$10 \mathrm{Ca}^{2+}+6 \mathrm{PO}_{4}{ }^{3-}+2 \mathrm{OH}^{-} \rightarrow \mathrm{Ca}_{10}\left(\mathrm{PO}_{4}\right)_{6}(\mathrm{OH})_{2}$

Preparation of LH: NCh was mixed with certain ion precursor buffer and 1\% wt/v aqueous acetic solution was used for dilution to the final concentration under constant stirring followed with tip sonication to completely and homogeneously re-disperse the mixture. Before tip sonication treatment, the dispersion was degassed via water bath ultrasonication and vacuum. Then the dispersion was placed in a cylindrical container and stored in a sealed environment with overdose of $25 \% \mathrm{wt} / \mathrm{v}$ aqueous ammonia for a period of time that depended on the volume (height) of the dispersion. As a result, a layered mineralized LH was obtained. For the LH with different shapes, the dispersion mixture was placed in a dialysis bag, and the bag was hung in an airtight container, as described before. For the preparation of sample for FT-IR, SEM and XPS analyses, the hydrogels were dehydrated (freeze drying) before testing. Before freeze drying, the hydrogels were washed by deionized water to remove free ions and water in the hydrogel was exchanged with tertiary butanol. The analysis methods can be seen in supplementary information.

Fabrication of nacre like HA-NCh plate (LP). To fabricate the bulk LP, the hydrogel was prepared in a polycarbonate mold. After the hydrogel was formed, the uniform part (with no layered mineralization structure) and the irregular part at the bottom of the gel were removed carefully. Then, the LH was taken out and immersed in deionized water to remove free ions. After thoroughly washed, the hydrogel was 
freeze dried to remove water. Eventually the dehydrated LH was transferred into the metal mold and hot pressed at $5 \mathrm{MPa}$ for $30 \mathrm{~min}$ and cooled down to room temperature. Then the nacre like LP was taken out and used for further tests.

\section{Declarations}

\section{Acknowledgements}

We are grateful for the financial support from the project funding from the Natural Science Foundation of Jiangsu Province (BK20190761), the Postgraduate Research \&Practice Innovation Program of Jiangsu Province (SJKY19_0893), and Project Funded by the National First-class Disciplines (PAFD). O.J.R acknowledges support by the Canadian Excellence Research Chair initiative and the Foundation for Innovation (CFI) as well as the European Research Council under the Horizon 2020 research and innovation program (ERC Advanced Grant Agreement No. 788489, “BioEICell”).

* Corresponding Author

\section{Author contributions}

J.X. and L.L. contributed equally to this work. J.X., Y.F., O.J.R. and Z.W. designed the research and participated in discussions and editing efforts; J.X. and L.L. carried out the experiments, analyzed the data and results, generated figures, and wrote and edited the initial manuscripts; J.Y., L.Z. and Y.Z. generated and analyzed the data and generated the figures; W.P. carried out the cytocompatibility tests; W.Y. produced the videos; L.B. and Q.Y. generated data and edited the manuscript.

\section{Competing interests}

The authors declare no competing interests.

\section{References}

[1] Yao, H. B., Fang, H. Y., Wang, X. H. \& Yu, S. H. Hierarchical assembly of micro-/nano-building blocks: bio-inspired rigid structural functional materials. Chem. Soc. Rev. 40, 3764-3785 (2011).

[2] Cheng, Q., Jiang, L. \& Tang, Z. Bioinspired layered materials with superior mechanical performance. Chem. Soc. Rev. 47, 1256-1266 (2014).

[3] Yao, H. B., Ge, J., Mao, L. B., Yan, Y. X. \& Yu, S. H. 25th anniversary article: artificial carbonate nanocrystals and layered structural nanocomposites inspired by nacre: synthesis, fabrication and applications. Adv. Mater. 26, 163-188 (2014).

[4] Monn, M. A., Vijaykumar, K., Kochiyama, S. \& Kesari, H. Lamellar architectures in stiff biomaterials may not always be templates for enhancing toughness in composites. Nat. Commun. 11, 1-12 (2020). 
[5] Barthelat, F., Yin, Z. \& Buehler, M. J. Structure and mechanics of interfaces in biological materials. Nat. Rev. Mater. 1, 1-16 (2016).

[6] Wang, J., Cheng, Q. \& Tang, Z. Layered nanocomposites inspired by the structure and mechanical properties of nacre. Chem. Soc. Rev. 41, 1111-1129 (2012).

[7] Liu, Y., Luo, D. \& Wang, T. Hierarchical structures of bone and bioinspired bone tissue engineering. Small 12, 4611-4632 (2016).

[8] Wei, Y. et al. Enamel Repair with Amorphous Ceramics. Adv. Mater. 32, 1907067 (2020).

[9] Deville, S., Saiz, E., Nalla, R. K. \& Tomsia, A. P. Freezing as a path to build complex composites. Science 311, 515-518 (2006).

[0] Zhao, H. \& Guo, L. Nacre-Inspired Structural Composites: Performance-Enhancement Strategy and Perspective. Adv. Mater. 29, 1702903 (2017).

[11] Checa, A. G., Cartwright, J. H. \& Willinger, M. G. The key role of the surface membrane in why gastropod nacre grows in towers. Proc. Natl. Acad. Sci. 106, 38-43 (2009).

[12] Tang, Z., Kotov, N. A., Magonov, S. \& Ozturk, B. Nanostructured artificial nacre. Nat. Mater. 2, 413-418 (2003).

[13] Finnemore, A. et al. Biomimetic layer-by-layer assembly of artificial nacre. Nat. Commun. 3, 1-6 (2012).

[14] Kim, Y. et al. Stretchable nanoparticle conductors with self-organized conductive pathways. Nature 500, 59-63 (2013).

[15] Bonderer, L. J., Studart, A. R. \& Gauckler, L. J. Bioinspired design and assembly of platelet reinforced polymer films. Science 319, 1069-1073 (2008).

[16] Laaksonen, P. et al. Genetic engineering of biomimetic nanocomposites: diblock proteins, graphene, and nanofibrillated cellulose. Angew. Chemie. Int. Ed. 50, 8688-8691 (2011).

[17] Das, P. et al. Nacre-mimetics with synthetic nanoclays up to ultrahigh aspect ratios. Nat. Commun. 6, 1-14 (2015).

[18] Zhu, B. et al. Hierarchical nacre mimetics with synergistic mechanical properties by control of molecular interactions in self-healing polymers. Angew. Chemie. Int. Ed. 54, 8653-8657 (2015).

[19] Hu, L. et al. Control of pore channel size during freeze casting of porous YSZ ceramics with unidirectionally aligned channels using different freezing temperatures. J. Eur. Ceram. Soc. 30, 3389-3396 (2010). 
[20] Munch, E. et al. Tough, bio-inspired hybrid materials. Science 322, 1516-1520 (2008).

[21] Bouville, F. et al. Strong, tough and stiff bioinspired ceramics from brittle constituents. Nat. Mater. 13, 508-514 (2014).

[22] Cheng, Q., Huang, C. \& Tomsia, A. P. Freeze casting for assembling bioinspired structural materials. Adv. Mater. 29, 1703155 (2017).

[23] Yan, X. et al. Nacre-mimic reinforced Ag@ reduced graphene oxide-sodium alginate composite film for wound healing. Sci. Rep. 7, 1-10 (2017).

[24] Wang, Y. et al. Artificial nacre from supramolecular assembly of graphene oxide. ACS Nano 12, 62286235 (2018).

[25] Li, Y. Q., Yu, T., Yang, T. Y., Zheng, L. X. \& Liao, K. Bio-inspired nacre-like composite films based on graphene with superior mechanical, electrical, and biocompatible properties. Adv. Mater. 24, 3426-3431 (2012).

[26] Zhang, C. J. et al. Nacre-inspired hydroxyapatite/chitosan layered composites effectively remove lead ions in continuous-flow wastewater. J. Hazard. Mater. 386, 121999 (2020).

[27] Morits, M. et al. Toughness and Fracture Properties in Nacre-Mimetic Clay/Polymer Nanocomposites. Adv. Funct. Mater. 27, 1605378 (2017).

[28] Liu, S., Li, K., Yao, F., Xu, L. \& Fu, G. Lanthanide ions-induced formation of hierarchical and transparent polysaccharide hybrid films. Carbohydrate Polymers 163, 28-33 (2017).

[29] Xie, W. et al. Extreme mechanical behavior of nacre-mimetic graphene-oxide and silk nanocomposites. Nano Lett. 18, 987-993 (2018).

[30] Hu, M. \& Mi, B. Layer-by-layer assembly of graphene oxide membranes via electrostatic interaction. J. Memb. Sci. 469, 80-87 (2014).

[31] Mao, L. B. et al. Synthetic nacre by predesigned matrix-directed mineralization. Science 354, 107-110 (2016).

[32] Pei, B., Wang, Z., Nie, J. \& Hu, Q. Highly mineralized chitosan-based material with large size, gradient mineral distribution and hierarchical structure. Carbohydr. Polym. 208, 336-344 (2019).

[33] Xu, C., Ouyang, C., Jia, R., Li, Y. \& Wang, X. Magnetic and optical properties of poly (vinylidene difluoride)/Fe304 nanocomposite prepared by coprecipitation approach. Journal of Applied Polymer Science 111, 1763-1768 (2009).

[34] Fan, Y., Saito, T. \& Isogai, A. Individual chitin nano-whiskers prepared from partially deacetylated achitin by fibril surface cationization. Carbohydr. Polym. 79, 1046-1051 (2010). 
[35] Liu, L. et al. High Axial Ratio Nanochitins for Ultrastrong and Shape-Recoverable Hydrogels and Cryogels via Ice Templating. ACS Nano 13, 2927-2935 (2019).

[36] Wang, D., Jang, J., Kim, K., Kim, J. \& Park, C. B. “Tree to Bone”: Lignin/Polycaprolactone Nanofibers for Hydroxyapatite Biomineralization. Biomacromolecules 20, 2684-2693 (2019).

[37] Song, S. et al. Ultrasmall graphene oxide modified with Fe304 nanoparticles as a Fenton-like agent for methylene blue degradation. ACS Appl. Nano Mater. 2, 7074-7084 (2019).

[38] Bai, H. et al. Bioinspired hydroxyapatite/poly (methyl methacrylate) composite with a nacre-mimetic architecture by a bidirectional freezing method. Adv. Mater. 28, 50-56 (2016).

[39] Liu, L. et al. Robust self-standing chitin nanofiber/nanowhisker hydrogels with designed surface charges and ultralow mass content via gas phase coagulation. Biomacromolecules 17, 3773-3781 (2016).

[40] Titze, T. et al. Transport in nanoporous materials including MOFs: The applicability of Fick's laws. Angew. Chem. Int. Edit. 54, 14580-14583 (2015).

[41] Himmelblau, D. M. Solubilities of Inert Gases in Water. $0^{\circ}$ C. to Near the Critical Point of Water. J. Chem. Eng. Data 5, 10-15 (1960).

[42] Lee, C., Fitzgerald, G., Planas, M. \& Novoa, J. J. Ionization of Bases in Water: Structure and Stability of the $\mathrm{NH}_{4}{ }^{+} \ldots \mathrm{OH}^{-}$Ionic Forms in Ammonia-Water Clusters. J. Phys. Chem. 100, 7398-7404 (1996).

[43] Gao, H. L.et al. Mass production of bulk artificial nacre with excellent mechanical properties. Nat. Commun. 8, 1-8 (2017).

[44] Wang, R. Z., Suo, Z., Evans, A. G., Yao, N. \& Aksay, I. A. Deformation mechanisms in nacre. J. Mater. Res. 16, 2485-2493 (2001).

[45] Bonfield, W., Wang, M. \& Tanner, K. E. Interfaces in analogue biomaterials. Acta Mater. 46, 2509-2518 (1998).

[46] Huang, S. J., Ke, J. H., Chen, G. J. \& Wang, L. F. One-pot synthesis of PDMAEMA-bound iron oxide nanoparticles for magnetofection. J. Mater. Chem. B 1, 5916-5924 (2013).

[47] Wang, X., Li, Y., Wei, J. \& De Groot, K. Development of biomimetic nano-hydroxyapatite/poly (hexamethylene adipamide) composites. Biomaterials 23, 4787-4791 (2002).

[48] Maté-Sánchez de Val, J. E. et al. Comparison of three hydroxyapatite/ $\beta$-tricalcium phosphate/collagen ceramic scaffolds: An in vivo study. J. Biomed. Mater. Res. A 102, 1037-1046 (2014). 
[49] Hu, Q., Li, B., Wang, M. \& Shen, J. Preparation and characterization of biodegradable chitosan/hydroxyapatite nanocomposite rods via in situ hybridization: a potential material as internal fixation of bone fracture. Biomaterials 25, 779-785 (2004).

\section{Figures}
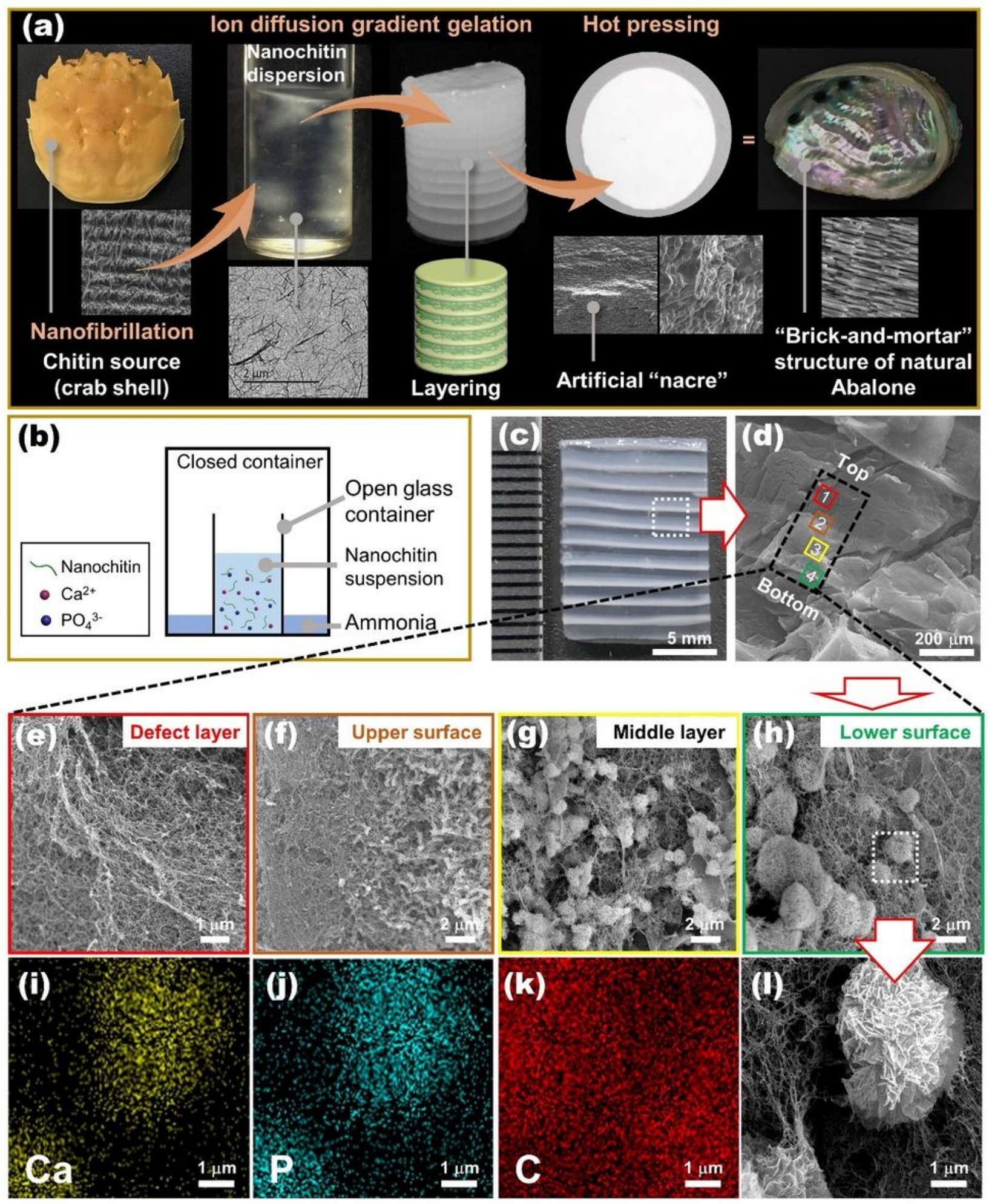

Figure 1 
LH synthesis and microstructure comprising layered HA-NCh hydrogels. (a) Schematic illustration of the development of LH structured as nacre-like sheets. (b) Schematic diagram of the device used for LH synthesis. (c) Photograph and (d) SEM image of LH showing a self-stratified layer structure. (e-h) Detailed structure of each LH layer, as shown in (d). (I) SEM of HA microspheres embedded in the NCh network and (i-k) EDS maps of (I).

(a)
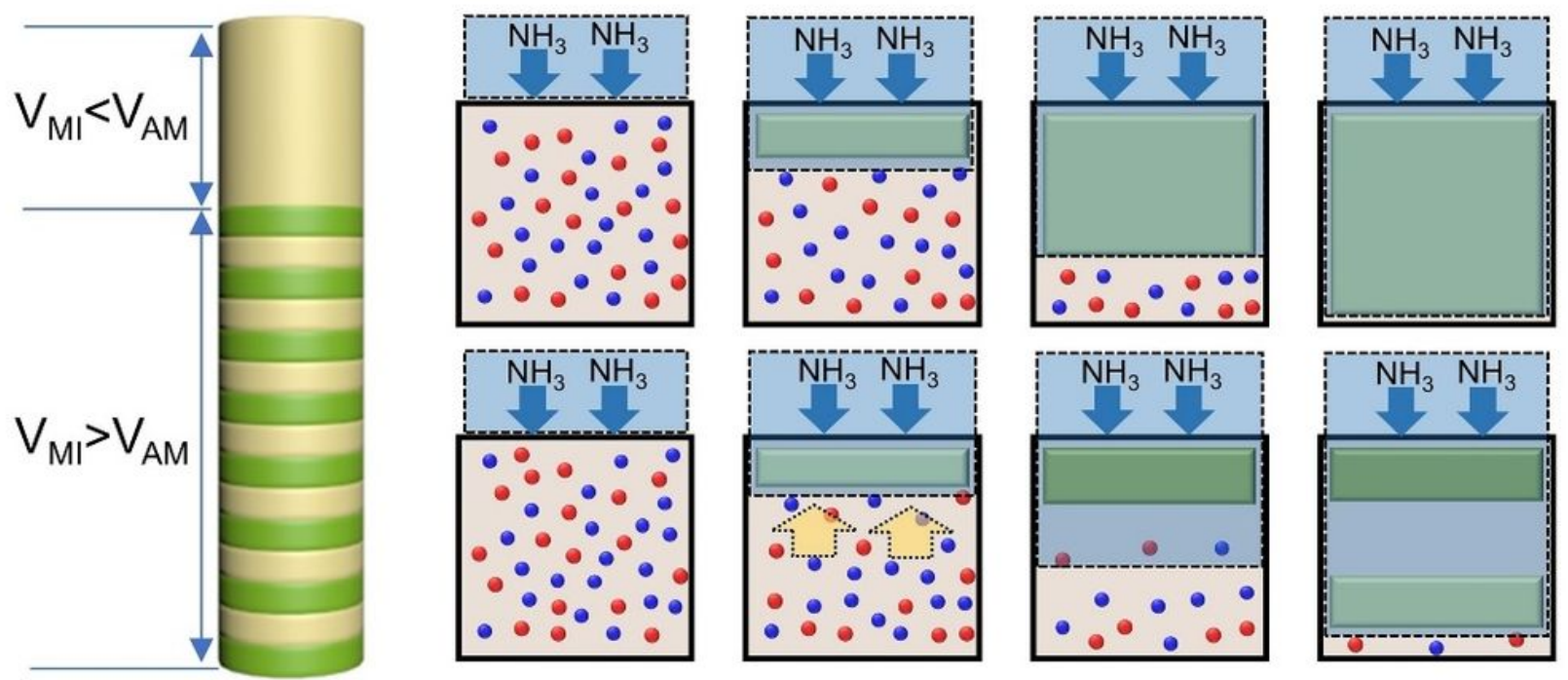

(b)
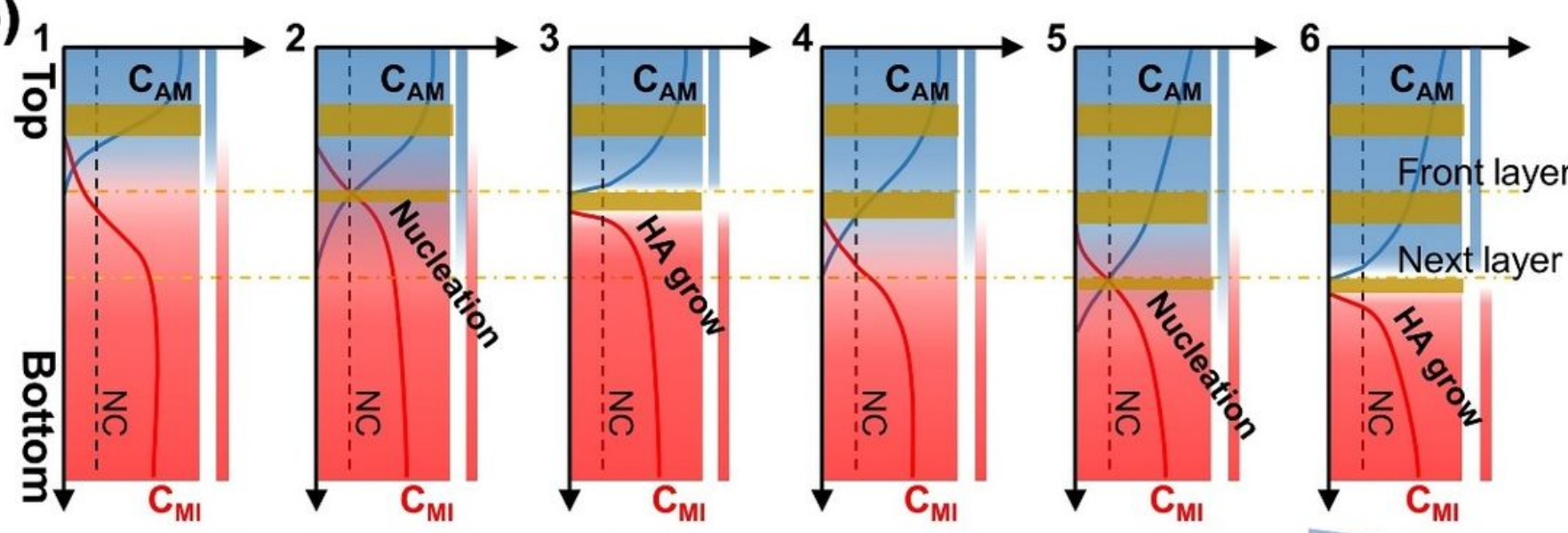

Time
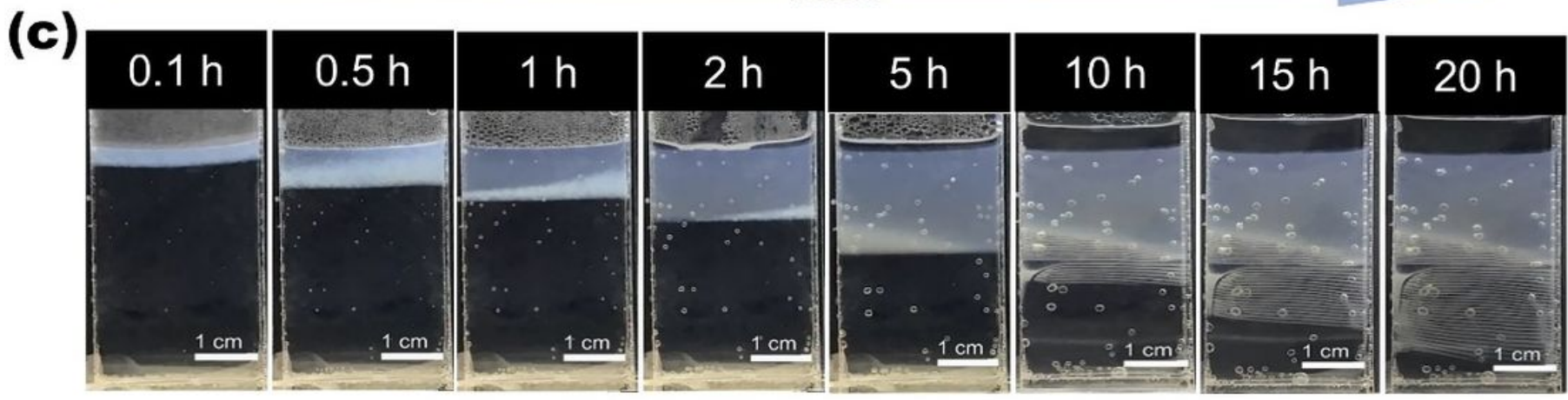

Figure 2 
Proposed mechanism leading to the formation of periodic layers of HA-NCh hydrogel (LH). (a) A uniform HA-NCh hydrogel is formed top-down when VMI < VAM (see sequence in the top row). Once VMI becomes greater than VAM, HA-NCh hydrogel self-assembles into self-stratified structures via ion diffusion (see sequence in the bottom row). The profiles in (b) are drawn to illustrate the suggested time evolution for self-stratification of HA layers in LH (see also Figure 3). Ammonia (AM) diffuses from top to bottom (b1). Nucleation occurs when CAM and CMI reach the nucleation concentration, NC (b2). Thereafter, mineral ions diffuse upward, to the nucleation site (forming HA), depleting ions and preventing nucleation (b3). With time, the concentration of mineral ions underneath the HA layer decreases, leading to $\mathrm{VMI}<\mathrm{VAM}$, which favors ammonia diffusion across the mineralized layer (b4), reaching another NC (b5) followed by the next HA layer (b6). (c) Sequence of photographs taken at given times, illustrating the LH formation process. Notation: VMI and VAM are the transfer rates of mineral and ammonia, respectively; CAM and $\mathrm{CMl}$ are the concentrations of ammonia and mineral ions, respectively. $\mathrm{NC}$ is the nucleation concentration.
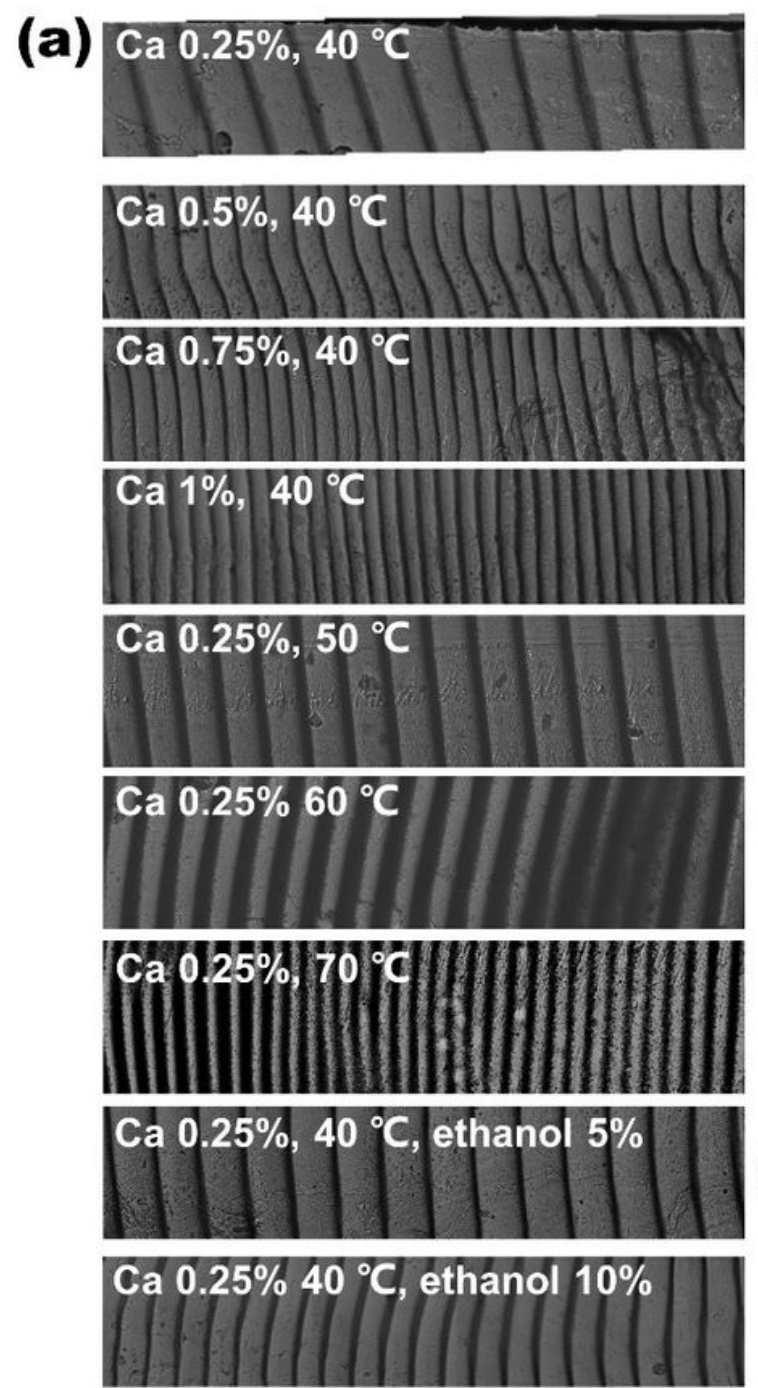

Ca $0.25 \%, 40{ }^{\circ} \mathrm{C}$, ethanol $20 \%$

$1 \mathrm{~mm}$
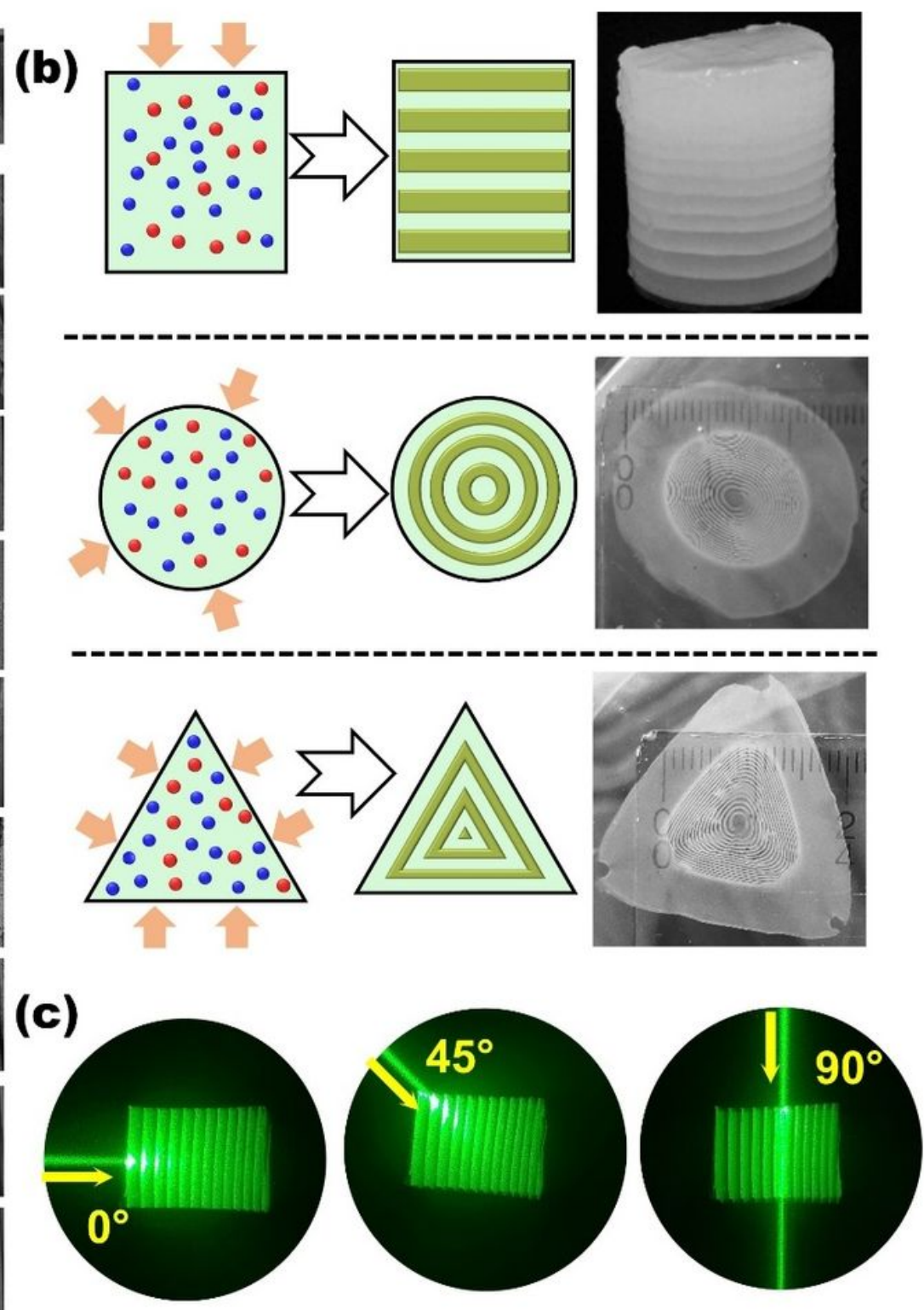


\section{Figure 3}

Structural regulation of LH: (a) Layer spacing control of LH by the selection of conditions (temperature, ion concentration and ethanol concentration). (b) Pattern control of LH depending on the direction of ammonia diffusion. (c) Light selective transmittance through one of the LH structures.
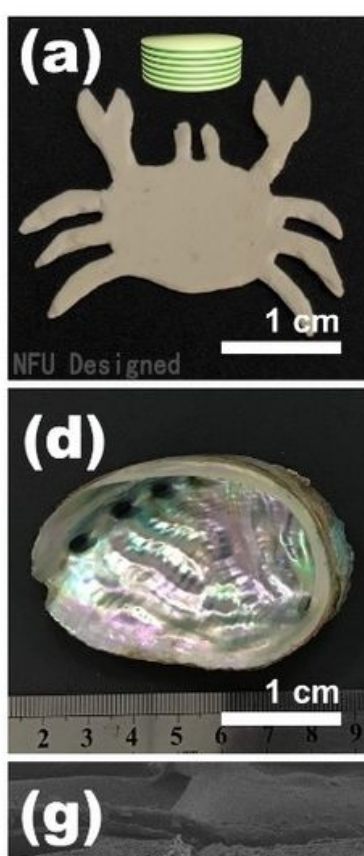
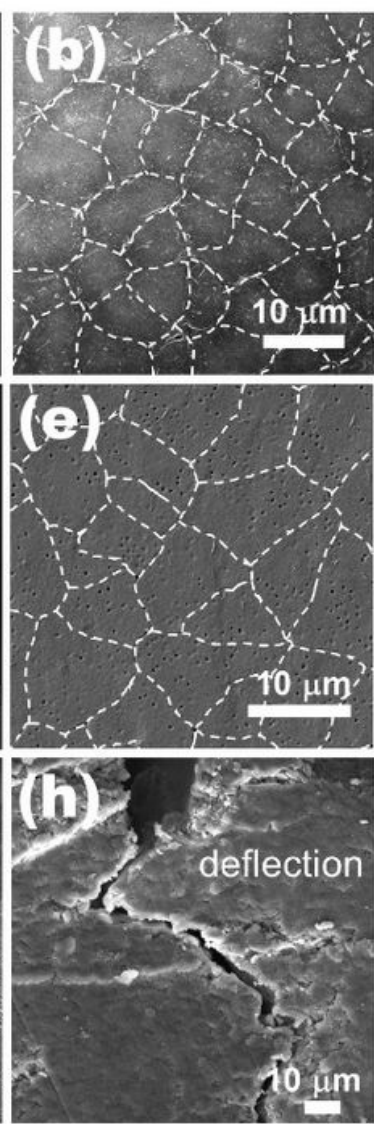
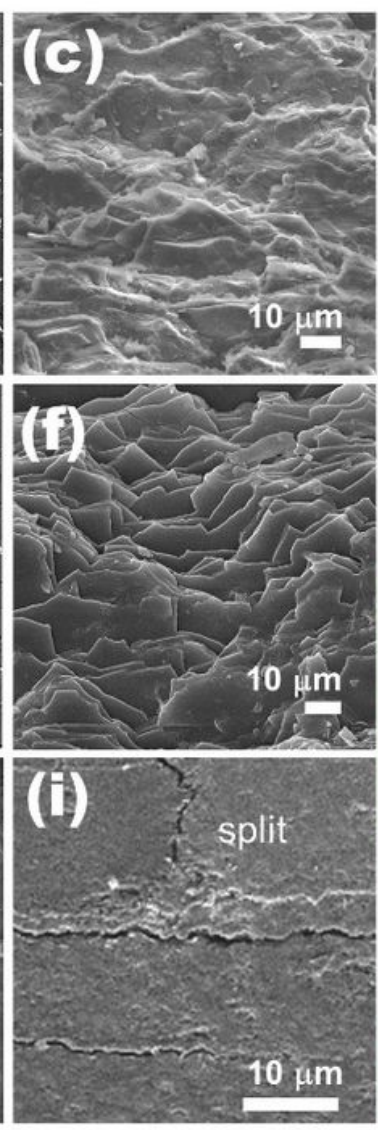

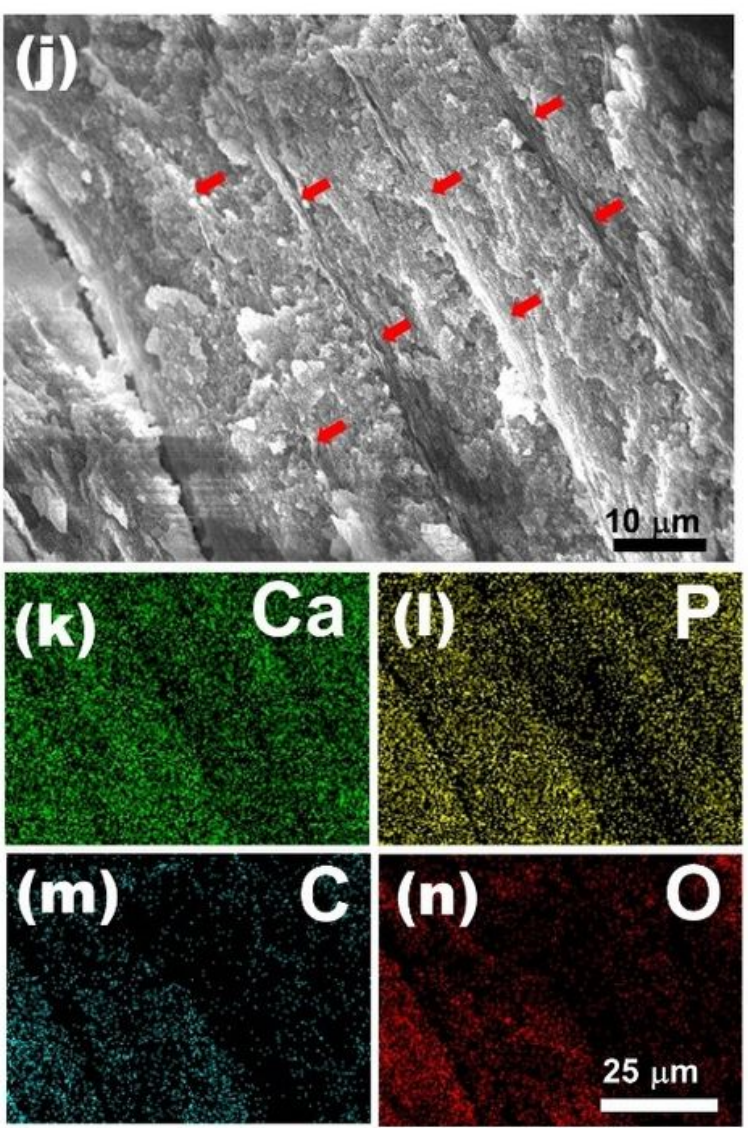

\section{Figure 4}

Structure of a solid plate (LP) prepared from layered HA-NCh: (a) Photograph of LP obtained upon hot pressing of a LH. (b) Voronoi pattern of the HA layer of LP observed by SEM. (c) SEM oblique fracture surface of LP. (d) Photograph of Abalone and its (e) Voronoi pattern (SEM) as well as (f) oblique fracture surface. (g) Profile of the fractured LP and $(\mathrm{h}, \mathrm{i})$ corresponding crack deflection and splitting between layers (magnified image of area in (g)). (j) Vertical fracture surface of LP (k-n). Surface element distribution (Ca, P, C, and O). 

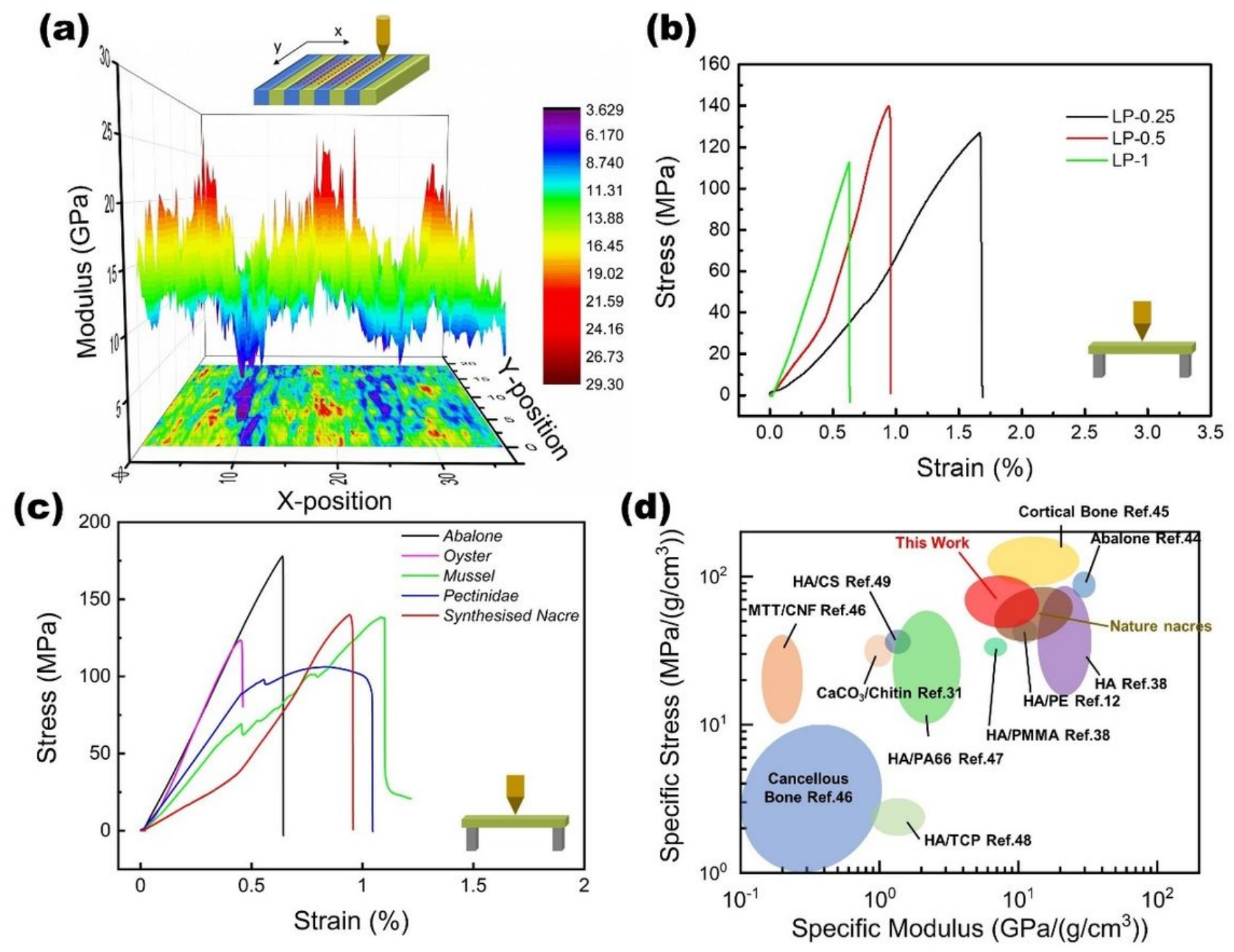

\section{Figure 5}

Mechanical properties of LP. (a) Surface modulus of the vertical fracture surface tested by nanoindentation. (b) Stress-strain profile of LP produced from HA at different $\mathrm{Ca} 2+$ concentrations, as indicated. (c) Stress-strain curve of natural pearls and LP (Ca2+ concentration of $0.5 \%$ ). (d) Specific fracture stress versus specific fracture modulus of $L P$, natural nacre and several artificial organic-mineral (hybrid) materials reported in the literature (see references) compared with the mechanical performance of our synthetic LP, shown in red color (see also Supplementary Fig. 15).

\section{Supplementary Files}

This is a list of supplementary files associated with this preprint. Click to download.

- supplementaryinformation.doc 
- supplementaryvideo1.mp4

- supplementaryvideo2.mp4

- supplementaryvideo3.mp4 